\title{
Serum Estradiol in Relation to Severity of Meibomian Gland Dysfunction in Postmenopausal Women
}

\author{
Priyadarshini Palaniyapphan¹, Mary Thomas², Gayatri Sundareswaran³, Sanjana Chilukuri \\ 1, 2, 3, 4 Department of Ophthalmology, Sri Ramachandra Institute of \\ Higher Education and Research, Chennai, Tamil Nadu, India.
}

\section{ABSTRACT}

\section{BACKGROUND}

Due to the higher prevalence of dry eye disease (DED) in postmenopausal women, it is essential to understand the effects of sex hormones on the ocular surface. This study was done to identify the association between serum concentration of 17 beta estradiol E2 and DED in postmenopausal women.

\section{METHODS}

Our study population comprised of 100 postmenopausal women who were segregated into two groups - 50 symptomatic participants in dry eye group and 50 asymptomatic participants in normal eye group (NEG), based on the dry eye questionnaire assessment. Other preliminary tests to detect DED and meibomian gland dysfunction (MGD) such as tear break up time (TBUT), meibum evaluation, ocular staining score (OSS) and Schirmer's test were performed. All participants underwent venous sampling for measurement of serum concentration of 17 beta estradiol E2.

\section{RESULTS}

$82 \%$ of participants of DEG belonged to older age group ( $>60$ years) on comparison with NEG (32\%). A shorter TBUT of 5.78 and 5.96 in DEG as compared to 13.44 and 13.08 in NEG were documented in right and left eyes respectively. Mean serum estradiol was significantly higher in DEG $(30.90 \mathrm{pg} / \mathrm{ml})$ than in NEG $(16.02 \mathrm{pg} / \mathrm{ml})$. With an increase in the severity of MGD stage, an analogous increase in serum estradiol was noted, which was statistically significant $(\mathrm{P}<0.001)$.

\section{CONCLUSIONS}

Higher levels of serum estradiol correspond with higher stages of MGD, suggesting that sex hormone induced alteration of meibomian gland morphology and function plays an important role in postmenopausal DED.

\section{KEY WORDS}

Dry Eye Disease, Meibomian Gland Dysfunction, 17 Beta Estradiol E2, Postmenopausal Women
Corresponding Author: Dr. Gayatri Sundareswaran, 9/15 Masilamani Road,Balaji Nagar, Chennai - 600014, Tamil Nadu, India.

E-mail: gayatri.sundareswaran@gmail.com

DOI: $10.14260 /$ jemds/2021/674

How to Cite This Article:

Palaniyapphan $P, \quad$ Thomas $M$, Sundareswaran G, et al. Serum Estradiol in relation to severity of meibomian gland dysfunction in postmenopausal Women. J Evolution Med Dent Sci 2021;10(38): 3322-3326 DOI:

10.14260/jemds/2021/674

Submission 06-07-2021,

Peer Review 24-08-2021,

Acceptance 31-08-2021,

Published 20-09-2021.

Copyright (C) 2021 Priyadarshini Palaniyapphan et al. This is an open access article distributed under Creative Commons Attribution License [Attribution 4.0 International (CC BY 4.0)] 


\section{BACKGROUND}

Dry eye disease is a multifactorial disease of the ocular surface that occurs in both genders and is more common among the elderly. Epidemiological data have shown that the prevalence of DED is higher among women and that has been party attributed to hormonal changes, especially post menopause. ${ }^{1}$ Sex hormones play a key role in maintaining ocular surface homeostasis and sex hormone receptors have been identified in ocular surface tissues. ${ }^{2}$

Meibomian gland dysfunction, one of the leading causes of evaporative dry eye is a chronic abnormality of the meibomian glands (MG) characterized by terminal duct obstruction and qualitative/quantitative changes in its secretion. ${ }^{3}$ The MG found in the upper and lower eyelids secretes lipids or meibum which forms the outermost layer of the tear film. With the up-phase of each blink, the upper lid draws the meibum over the anterior surface of the tear film, thus retarding tear film evaporation. With the down-phase of the blink, the meibum is returned to the marginal reservoir. ${ }^{2}$ With dysfunction of the MG, altered lipid secretion contributes to an unstable tear film, which results in symptoms of ocular discomfort. This tear film instability can be measured clinically as tear break up time. ${ }^{4}$

MGD may be classified as low delivery or high delivery types, the former being more common. Low delivery MGD is further categorized as obstructive or hyposecretory subtypes. Obstructive MGD being the most prevalent form of MGD is characterized by hyperkeratinisation of meibomian ducts and increased viscosity of the meibomian oil. ${ }^{3}$ Changes in the chemical composition of the meibum are responsible for increased viscosity, and this can be assessed clinically as meibum expressibility and quality. ${ }^{2}$

Pathophysiology of MGD is characterized by keratinization of MG orifices, tear film instability and hyperosmolarity. This further leads to stasis of the meibum causing bacterial proliferation and release of lipases and esterases, which results in inflammation and increased meibum viscosity. These changes are accompanied by further MG blockade and atrophy as well as loss of goblet cells, blocking the delivery of mucin to the ocular surface and contributing to the development of DED. In this way, the pathophysiology of MGD and DED form a double vicious circle. ${ }^{3}$ All these deviations are influenced by external factors such age, allergy, contact lens use, systemic drugs and hormonal imbalance among others.

DED in postmenopausal women has been analyzed before and conflicting results have been reported concerning the effects of high and low serum estrogen. The influence of serum androgen and hormone replacement therapy (HRT) have also been extensively studied. ${ }^{1}$ This study aims to understand the association between serum concentration of 17 beta estradiol (E2) and DED in postmenopausal women.

\section{METHODS}

This is a cross-sectional study performed in a tertiary hospital from February 2019 to October 2019. A study population of 100 postmenopausal women above the age of 50 with cessation of menses for a minimum period of 1 year were included. Participants with history of prior hysterectomy/oophorectomy, autoimmune disorders, prior ocular surgery, HRT, immunosuppressants, antidepressants were excluded from our study. Based on symptom assessment by the ocular surface disease index (OSDI) questionnaire, participants who secured a score above 12 were assigned in dry eye group (DEG), and those below 12 were assigned in normal eye group (NEG).

\section{Sample Size}

Sample size was calculated with nMaster software version 2.0; with sample correlation coefficient as 0.866 and population correlation coefficient as 0.5 , alpha error of $5 \%$ and power of the study as $99 \%$, the minimum sample size calculated for each arm was 34 . Hence, sample size of 68 was calculated, which further included a total of 100 participants.

The study proposal was approved by the Institutional Ethics Committee and was conducted according to the tenets of declaration of Helsinki. Participants and attenders were explained about the study and written informed consent was obtained.

The participants underwent symptom assessment with OSDI questionnaire. Slit lamp examination to assess the meibum quality and expressibility of the central ten MG of lower lid was performed. MG secretions were evaluated according to the scoring proposed by Foulks and Bron. ${ }^{2}$

a) Meibum expressibility - was scored from 0 to 3 based on the amount of pressure required to express the $M G$ secretion: 0 - minimal; 1 - mild; 2 - moderate; 3 - heavy pressure.

b) Meibum quality - was scored from 0 to 4 based on the colour and viscosity: 0 - clear oil; 1 - cloudy secretion; 2 granular secretion; 3 - solid and paste-like meibum; 4 nil meibum expressed.

c) Dry eye tests - such as TBUT, Schirmer's test ${ }^{4}$ and ocular staining score (OSS) ${ }^{5}$ were measured.

d) MGD staging- Severity of MGD was graded by the tear film and ocular surface (TFOS) workshop devised staging system based on meibum quality, expressibility, patient symptoms and corneal staining. ${ }^{6}$

e) Serum concentration of E2- $2 \mathrm{ml}$ of venous blood was collected from the participants at 8 am after overnight fasting and the sample was sent for enzyme linked immunosorbent assay (ELISA) analysis of E2.

\section{Statistical Analysis}

The collected data was analyzed using IBM. Statistical Package for Social Sciences (SPSS statistics software 23.0 version). To find the significant difference between the bivariate samples in independent groups (DEG and NEG), the Mann-Whitney U test was used. To determine the significance in categorical data, chi-square test was used. The probability value $<0.01$ was considered significant.

\section{RESULTS}

\section{Age Distribution}

$82 \%$ of participants in the DEG belonged to a higher age group (> 60 years) as compared to $32 \%$ in the NEG (Table 1) 


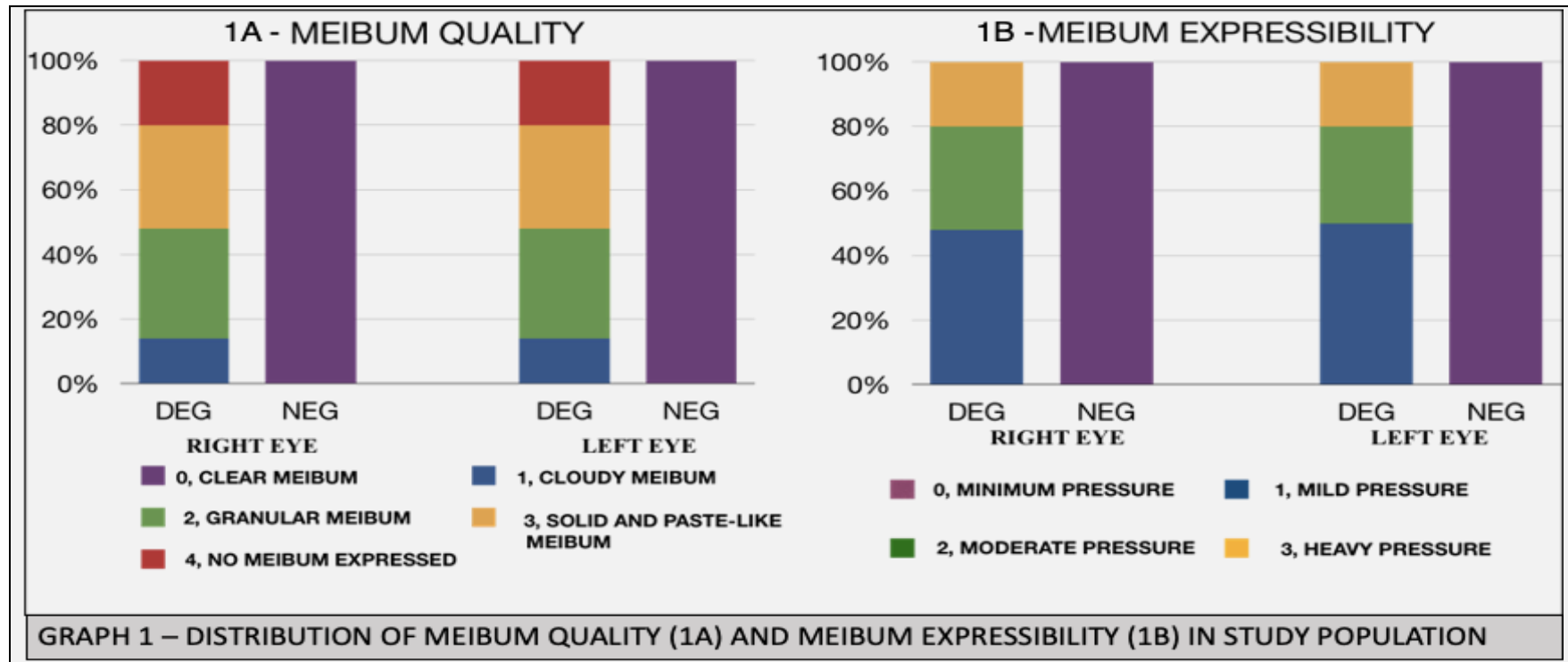

Graph 1. Distribution of Meibum Quality (1A) and Meibum Expressibilty (1B) in Study Population

\begin{tabular}{|c|c|c|c|}
\hline Age (in Years) & DEG & NEG & P Value \\
\hline $51-55$ & $1(2 \%)$ & $12(24 \%)$ & \multirow{6}{*}{$<0.001$} \\
\hline $56-60$ & $8(16 \%)$ & $22(44 \%)$ & \\
\hline $60-65$ & $16(32 \%)$ & $14(28 \%)$ & \\
\hline $65-70$ & $22(44 \%)$ & $2(4 \%)$ & \\
\hline$>$ & $3(6 \%)$ & $0(0 \%)$ & \\
\hline Total & $50(100 \%)$ & $50(100 \%)$ & \\
\hline \multicolumn{4}{|c|}{ Table 1. Age Distribution of Study Population } \\
\hline DEG- Dry eye group & - Normal eye & & \\
\hline
\end{tabular}

\begin{tabular}{|cccc|}
\hline OSDI Score & DEG & NEG & P Value \\
Normal $(0-12)$ & $0(0 \%)$ & $50(100 \%)$ & \\
Mild $(13-22)$ & $24(48 \%)$ & $0(0 \%)$ & \\
Moderate $(23-33)$ & $16(32 \%)$ & $0(0 \%)$ & $<0.001$ \\
Severe $(33-100)$ & $10(20 \%)$ & $0(0 \%)$ & \\
Total & $50(100 \%)$ & $50(100 \%)$ & \\
\hline Table 2. Prevalence And Severity Of Dry Eye By Osdi Questionnaire \\
\hline DEG- Dry eye group; NEG - Normal eye group: OSDI - Ocular Surface Disease \\
Index
\end{tabular}

\section{Meibum Quality and Expressibility}

All 50 participants in NEG had normal meibum quality and expressibility; participants of DEG had altered meibum scores as shown in graph $1(\mathrm{P}<0.001)$.

\section{Dry Eye Tests}

Participants of DEG had raised ODSI scores, shorter TBUT, elevated OSS and unremarkable Schirmer's when compared to NEG in both eyes. (Table 3)

\begin{tabular}{|c|c|c|c|c|}
\hline Test & Group & Mean Value & S.D. & P Value \\
\hline \multirow{2}{*}{ OSDI score } & DEG & 28.08 & 15.88 & \multirow{2}{*}{$<0.001$} \\
\hline & NEG & 7.14 & 1.70 & \\
\hline \multirow{2}{*}{ TBUT right eye } & DEG & 5.78 & 1.57 & \multirow{2}{*}{$<0.001$} \\
\hline & NEG & 13.44 & 1.43 & \\
\hline \multirow{2}{*}{ TBUT left eye } & DEG & 5.96 & 1.54 & \multirow{2}{*}{$<0.001$} \\
\hline & NEG & 13.08 & 1.28 & \\
\hline \multirow{2}{*}{ OSS right eye } & DEG & 6.96 & 1.92 & \multirow{2}{*}{$<0.001$} \\
\hline & NEG & 0.60 & 0.49 & \\
\hline \multirow{2}{*}{ OSS left eye } & DEG & 6.76 & 1.82 & \multirow{2}{*}{$<0.001$} \\
\hline & NEG & 0.54 & 0.50 & \\
\hline \multirow{2}{*}{ Schirmer's test right eye } & DEG & 20.20 & 3.19 & \multirow{2}{*}{0.3} \\
\hline & NEG & 21.20 & 4.80 & \\
\hline \multirow{2}{*}{ Schirmer's test left eye } & DEG & 19.80 & 3.34 & \multirow{2}{*}{0.3} \\
\hline & NEG & 23.70 & 4.38 & \\
\hline \multicolumn{5}{|c|}{ Table 3. Dry Eye Tests in Study Population } \\
\hline
\end{tabular}

\section{Serum Concentration of Estradiol}

Mean value of E2 was $30.90( \pm 3.11) \mathrm{pg} / \mathrm{ml}$ in DEG and 16.02 $( \pm 1.32) \mathrm{pg} / \mathrm{ml}$ in NEG $(\mathrm{P}<0.001)$.

\section{Comparison of MGD Stages with E2}

As per the staging devised by TFOS, 7 in our study the mean value of serum E2 increases as the severity of MGD increases (Table 4).

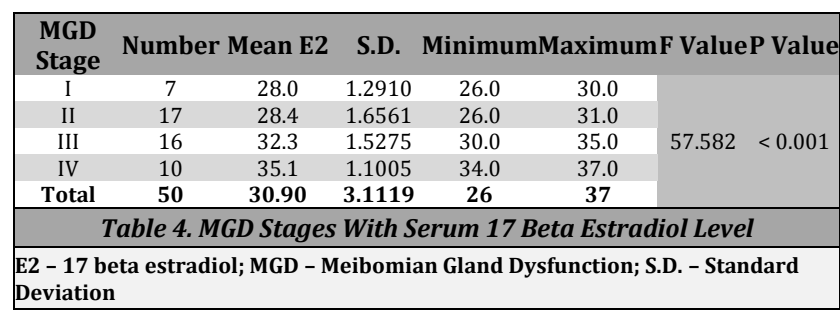

\section{DISCUSSION}

DED is a chronic and progressive condition of the ocular surface with a prevalence that varies from 5 to $50 \%$, highest amongst Asians. ${ }^{8}$ The prevalence of DED increases with age, and is more common in women, especially post menopausal.9,10 Other risk factors for DED are hormonal dysfunction, contact lens use,11 refractive surgery, autoimmune disorders and systemic drug effects. ${ }^{12}$

DED has been classified by dry eye workshop (DEWS) based on etiology into aqueous tear deficiency due to inadequate production of tears by lacrimal gland, and evaporative tear deficiency predominantly due to MGD causing tear film instability. ${ }^{13}$ These two types of DED are not mutually exclusive and have overlapping signs and symptoms. MGD is a leading cause of DED with a prevalence of $60-69 \%$ in Asians above the age of $40 .{ }^{14}$ It is characterized by obstruction of MG orifices and qualitative/quantitative alterations in the secretions. Patients with obstructive MGD have terminal duct obstruction that could be due to cicatricial or non-cicatricial etiologies. 
Predisposing factors such as older age, female gender and hormonal disturbances influence the obstructive process. The consequences of MGD are tear film instability, hyperosmolarity, ocular surface inflammation and symptoms of dry eye. ${ }^{6}$

The expressed meibomian oil from the glands is an amalgam of the MG secretions and keratinized epithelial debris. This mixture of nonpolar and polar lipids is in a liquid state at core lid temperature of about 37 degrees celsius. With each blink, the oil is delivered from the lid margin reservoir to the tear film and helps prevent evaporation of tears. ${ }^{2}$ In normal subjects, clear oil can be freely expressed with minimal digital pressure over the eyelid margin. Various grading scales have been described to assess the characteristics of the meibum. The expressibility of meibum is assessed based on the amount of pressure required, and the quality of the expressed meibum is graded based on the colour and viscosity of the oil.15

TBUT is the time interim between the last complete lid closure and the earliest appearance of a fluorescein break spot on the corneal surface. ${ }^{16}$ This is a crucial test for identifying evaporative dry eye, as an inadequate tear film lipid layer in MGD exhibits a shorter TBUT.15 In our study, patients with symptomatic dry eye had a significantly shorter TBUT $(\mathrm{P}<0.001)$.

Among the several validated questionnaires for dry eye, we chose the OSDI as it has high sensitivity and specificity to distinguish between normal and dry eye participants, while being concise and convenient. 10 OSDI scores range from 0 100 , where $<12$ is considered normal (NEG) and higher scores (DEG) were graded on a scale as mild, moderate and severe dry eye. ${ }^{7}$ Ocular surface staining score with application of fluorescein and Lissamine green were documented as OSS ranging from $0-12.5$ DEG had a mean score of 6.96 and 6.76, while NEG had a score of 0.60 and 0.54 in the right and left eye respectively $(\mathrm{P}<0.001)$.

Due to the female preponderance of DED, especially in postmenopausal women, sex hormones have been implicated in its pathogenesis. Receptor proteins for estrogen and androgen have been identified in the conjunctiva, cornea, lacrimal and meibomian glands.17,18 Schirra et al. also demonstrated the presence of steroidogenic enzyme mRNAs in the ocular surface required for the intracrine synthesis and metabolism of sex steroids. ${ }^{19}$

E2 is a steroid hormone produced primarily by the ovaries during the reproductive lifespan. It acts via binding to specific receptors in estrogen responsive tissues. Average premenopausal serum E2 ranges from 30 to $400 \mathrm{pg} / \mathrm{ml}$, which declines to 0 to $30 \mathrm{pg} / \mathrm{ml}$ after menopause. ${ }^{14}$ It has been postulated that estradiol negatively influences lipid secretion in the MG, by attenuation of lipid synthesis and enhancement of lipid catabolism.20,21 In our study, the mean value of estradiol was 16.02 in NEG and 30.9 in DEG. (P < 0.001). With a rise in the severity of MGD, a corresponding rise in the level of E2 was documented (Table 5). Higher levels of E2 were associated with more alterations of meibum quality/expressibility, higher OSDI and OSS scores, shorter TBUT and unremarkable Schirmer's, suggestive of an increasing severity of evaporative DED (Table 3). In a similar study by Golebiowski et al. raised serum E2 level was associated with worse meibum quality, increased capping of MG orifices, shorter TBUT and pronounced corneal staining. ${ }^{22}$
This corresponds to other reports of high serum estradiol implicated as a risk factor for DED.1,14 In parallel reports, the subjective symptoms of DED, tear production and tear stability were recognized to follow hormonal fluctuations during the different phases of the menstrual cycle. The follicular phase of the cycle during which estrogen is at its peak was found to have shorter TBUT, suggestive of estrogen's antisebaceous effect on MG.23,21,20 However, an analysis by Gagliano et al. demonstrated lower levels of E2 in postmenopausal women with dry eye, when compared to controls. ${ }^{18}$

To further substantiate our study's outcome, supportive literature on the association of dry eye and HRT intake may be cited. Estradiol is a major component of HRT which is used therapeutically by postmenopausal women to alleviate symptoms of menopause. Postmenopausal women on HRT had higher prevalence of DED than those who were not on HRT. ${ }^{24}$ The risk of DED was directly proportional to the duration of HRT use.25 Versura et al. stated that while high level of serum estrogen can lead to MGD, low level of serum androgen is also a risk factor for DED. ${ }^{20}$ Androgens have a prosebaceous effect on MG function and exercise a protective role on the ocular surface..$^{9,26}$

Our study confirms the association between raised levels of serum estradiol and MGD in postmenopausal women. Elevated E2 has implications on the MG secretions leading to evaporative DED. Further studies on infrared analysis of MG architecture, lipid layer thickness and tear osmolarity measurement may give additional information on the hormonal effects on the glands.

\section{CONCLUSIONS}

We conclude that serum concentrations of 17 beta estradiol were elevated in postmenopausal women with DED. Higher level of E2 corresponds with higher grade of severity of MGD with altered meibum score, shorter TBUT and higher OSS. Hence, we suggest that raised serum estrogen levels have implications on meibomian gland morphology and function leading to evaporative DED in postmenopausal women.

Data sharing statement provided by the authors is available with the full text of this article at jemds.com.

Financial or other competing interests: None.

Disclosure forms provided by the authors are available with the full text of this article at jemds.com.

\section{REFERENCES}

[1] Shen G, Ma X. High levels of $17 \beta$-Estradiol are associated with increased matrix Metalloproteinase-2 and Metalloproteinase- 9 activity in tears of postmenopausal women with dry eye. J Ophthalmol 2016;2016:2415867.

[2] Foulks GN, Bron AJ. Meibomian gland dysfunction: a clinical scheme for description, diagnosis, classification, and grading. The Ocular Surface 2003;1(3):107-26.

[3] Baudouin C, Messmer EM, Aragona P, et al. Revisiting the vicious circle of dry eye disease: a focus on the 
pathophysiology of meibomian gland dysfunction. $\mathrm{Br} \mathrm{J}$ Ophthalmol 2016;100(3):300-6.

[4] Zeev MS-B, Miller DD, Latkany R. Diagnosis of dry eye disease and emerging technologies. Clin Ophthalmol 2014;8:581-90.

[5] Whitcher JP, Shiboski CH, Shiboski SC, et al. A simplified quantitative method for assessing keratoconjunctivitis sicca from the Sjögren's Syndrome International Registry. Am J Ophthalmol 2010;149(3):405-15.

[6] Nichols KK, Foulks GN, Bron AJ, et al. The International Workshop on Meibomian Gland Dysfunction: Executive Summary. Invest Ophthalmol Vis Sci 2011;52(4):1922-9.

[7] Grubbs JR, Tolleson-Rinehart S, Huynh K, et al. A review of quality of life measures in dry eye questionnaires. Cornea 2014;33(2):215-8.

[8] Stapleton F, Alves M, Bunya VY, et al. TFOS DEWS II epidemiology report. The Ocular Surface 2017;15(3):334-65.

[9] Garcia-Alfaro P, Bergamaschi L, Marcos C, et al. Prevalence of ocular surface disease symptoms in periand postmenopausal women. Menopause $\mathrm{N} Y \mathrm{~N}$ 2020;27(9):993-8.

[10] Matossian C, McDonald M, Donaldson KE, et al. Dry eye disease: consideration for women's health. J Womens Health 2019;28(4):502-14.

[11] Schaumberg DA, Nichols JJ, Papas EB, et al. The International Workshop on Meibomian Gland Dysfunction: Report of the Subcommittee on the Epidemiology of and Associated Risk Factors for, MGD. Invest Ophthalmol Vis Sci 2011;52(4):1994-2005.

[12] Lemp MA, Crews LA, Bron AJ, et al. Distribution of aqueous-deficient and evaporative dry eye in a clinicbased patient cohort: a retrospective study. Cornea 2012;31(5):472-8.

[13] DEWS Definition and Classification. The definition and classification of dry eye disease: report of the Definition and Classification Subcommittee of the International Dry Eye WorkShop (2007). Ocul Surf 2007;5(2):75-92.

[14] Ablamowicz AF, Nichols JJ, Nichols KK. Association between serum levels of testosterone and estradiol with meibomian gland assessments in postmenopausal women. Invest Ophthalmol Vis Sci 2016;57(2):295-300.
[15] Tomlinson A, Bron AJ, Korb DR, et al. The international workshop on meibomian gland dysfunction: report of the diagnosis subcommittee. Invest Ophthalmol Vis Sci 2011;52(4):2006-49.

[16] Li J, Ma J, Hu M, et al. Assessment of tear film lipid layer thickness in patients with Meibomian gland dysfunction at different ages. BMC Ophthalmol 2020;20(1):394.

[17] Rocha EM, Wickham LA, da Silveira LA, et al. Identification of androgen receptor protein and 5alphareductase mRNA in human ocular tissues. $\mathrm{Br} J$ Ophthalmol 2000;84(1):76-84.

[18] Gagliano C, Caruso S, Napolitano G, et al. Low levels of 17 - $\beta$-oestradiol, oestrone and testosterone correlate with severe evaporative dysfunctional tear syndrome in postmenopausal women: a case-control study. British Journal of Ophthalmology 2014;98(3):371-6.

[19] Schirra F, Suzuki T, Dickinson DP, et al. Identification of steroidogenic enzyme mRNAs in the human lacrimal gland, meibomian gland, cornea, and conjunctiva. Cornea 2006;25(4):438-42.

[20] Versura P, Giannaccare G, Campos EC. Sex-steroid imbalance in females and dry eye. Curr Eye Res 2015;40(2):162-75.

[21] Sriprasert I, Warren DW, Mircheff AK, et al. Dry eye in postmenopausal women: a hormonal disorder. Menopause 2016;23(3):343-51.

[22] Golebiowski B, Badarudin N, Eden J, et al. Does endogenous serum oestrogen play a role in meibomian gland dysfunction in postmenopausal women with dry eye? Br J Ophthalmol 2017;101(2):218-22.

[23] Versura P, Fresina M, Campos EC. Ocular surface changes over the menstrual cycle in women with and without dry eye. Gynecol Endocrinol 2007;23(7):385-90.

[24] Chia EM, Mitchell P, Rochtchina E, et al. Prevalence and associations of dry eye syndrome in an older population: the Blue Mountains Eye Study. Clin Experiment Ophthalmol 2003;31(3):229-32.

[25] Schaumberg DA, Buring JE, Sullivan DA, et al. Hormone replacement therapy and dry eye syndrome. JAMA 2001;286(17):2114-9.

[26] Sullivan DA, Sullivan BD, Evans JE, et al. Androgen deficiency, Meibomian gland dysfunction and evaporative dry eye. Ann N Y Acad Sci 2002;966:211-22. 